\title{
Effects of FTY720 (Fingolimod) on Proliferation, Differentiation, and Migration of Brain-Derived Neural Stem Cells
}

\author{
Botao Tan, ${ }^{1}$ Zeruxin Luo, ${ }^{1}$ Yan Yue, ${ }^{1,2}$ Yuan Liu, ${ }^{3}$ Li Pan, ${ }^{1}$ Lehua Yu, ${ }^{1,4}$ and Ying Yin \\ ${ }^{1}$ Department of Rehabilitation Medicine, The 2nd Affiliated Hospital of Chongqing Medical University, Chongqing 400010, China \\ ${ }^{2}$ Physical Examination Center, Chongqing General Hospital, Chongqing, China \\ ${ }^{3}$ The 3rd Department of Research Institute of Surgery, Daping Hospital, The Third Military Medical University, \\ State Key Laboratory of Trauma, Burns and Combined Injury, Chongqing, China \\ ${ }^{4}$ Kuanren Rehabilitation Hospital, The 2nd Affiliated Hospital of Chongqing Medical University, Chongqing, China
}

Correspondence should be addressed to Ying Yin; iyinying@hotmail.com

Received 7 May 2016; Revised 31 August 2016; Accepted 20 September 2016

Academic Editor: Laura Lasagni

Copyright (C) 2016 Botao Tan et al. This is an open access article distributed under the Creative Commons Attribution License, which permits unrestricted use, distribution, and reproduction in any medium, provided the original work is properly cited.

Insufficient proliferation, differentiation, and migration are the main pitfalls of neural stem cells (NSCs) in reparative therapeutics for the central nervous system (CNS) diseases. The potent lipid mediator sphingosine-1-phosphate (S1P) regulates cells' biological behavior broadly in the CNS. However, the effects of activating S1P on NSCs are not quite clear. In the current study, FTY720 (Fingolimod), an analog of S1P, was employed to induce the proliferation, differentiation, and migration of cultured brain-derived NSCs. The results indicated that proliferation and migration ability of NSCs were promoted by FTY720. Though we observed no obvious neuron prefers differentiation of NSCs, there were more protoplasmic astrocytes developed in the presence of certain concentration of FTY720. This work gives more comprehensive understanding of how FTY720 affects NSCs.

\section{Introduction}

Proliferation, differentiation, and migration properties of neural stem cells (NSCs) provide a promising future in repairing the injured or degenerated disorders of central nervous system [1]. However, the limitations of NSCs such as insufficient proliferation, failing of differentiating towards desired cell types, and not being able to migrate efficiently make it still a long way of utilizing them in clinical practice [2]. It was pointed out that biological behavior of NSCs was controlled by various intrinsic characteristics and extrinsic signals [3-5]. Among these, lipid mediators would be one of the undervalued candidates in regulating NSCs proliferation, migration, and differentiation.

Potent lipid mediator sphingosine-1-phosphate (S1P) as one of transduces intracellular signals plays a critical role in cells' biological behavior in the CNS by activating sphingosine-1-phosphate receptors (S1PRs) [6-8]. S1PRs participate in the anti-inflammatory process of microglia and proliferation of astrocytes, but their roles on NSCs have seldom been reported $[9,10]$. Evidence showed that S1PRs are expressed in NSCs and the activation of S1PRs, especially S1P1, would regulate the migration and angiogenesis of NSCs $[11,12]$. With the booming number of studies on stem cell transplantation or endogenous NSCs in CNS diseases, it is pending to demonstrate the role of S1PRs/S1P in regulating NSCs in detail.

In the present study, we employed a structural analog to S1P: FTY720, which is known as an oral drug for multiple sclerosis, to intervene in primary cultured NSCs [13]. We found out that migration and proliferation of NSCs were positively affected by FTY720. Though no significant differences of neuron preferring differentiation were found, there were more protoplasmic astrocytes developed in the presence of certain concentration of FTY720. This study gives more understanding of FTY720 in biological behavior of NSCs. 


\section{Materials and Methods}

2.1. Culture of Neural Stem Cells. All experiments were approved by the Animal Care and Experimental Committee of Chongqing Medical University. NSCs were collected from embryonic 13.5 days (E13.5) of Sprague-Dawley rats as described previously $[14,15]$. Briefly, the telencephalon was rapidly dissected and mechanically dissociated into singlecell suspension in tubes containing $0.25 \%$ trypsin. Single-cell suspension was then transferred to growth medium consisting of NB (Gibco, USA) + 2\% B27 (Gibico, USA) supplemented with $20 \mathrm{ng} / \mathrm{mL}$ human recombinant basic fibroblast growth factor (bFGF; Invitrogen, USA) and $20 \mathrm{ng} / \mathrm{mL}$ epidermal growth factor (EGF; Invitrogen, USA). This also referred to basic condition medium. Then, cells were cultured at $37^{\circ} \mathrm{C}$ under a humidified atmosphere containing $5 \% \mathrm{CO}_{2}$. Half of the medium was replaced every other day. Secondary or tertiary neurospheres were used for subsequent experiments.

For Brd-U incorporation, NSCs were incubated in growth medium containing $10 \mathrm{mM}$ Brd-U (Sigma, USA) for 18 hours before immunocytochemical analysis.

2.2. FTY720 Intervention. To examine the effect of FTY720 (FTY720-P: FTY720 phosphate: Cayman, USA) [16], NSCs were divided into four groups according to the concentration of FTY-720 ( $0 \mathrm{nM}, 1 \mathrm{nM}, 10 \mathrm{nM}$, and $100 \mathrm{nM}$, diluted in DMSO) in basic culture medium within (for differentiation) or without (for CCK-8 testing and the sphere number counting) $10 \%$ fetal bovine serum (HyClone, Thermo, USA). $0 \mathrm{nM}$ FTY720 was recognized as the control group. The differentiated cells were fixed and immunochemically stained with specific markers of neurons, astrocytes, and oligodendrocytes.

2.3. CCK-8 Assay. Cell counting kit-8 (CCK-8; Dojindo, Japan) was processed following the instructions, and passage cells of $100 \mu \mathrm{L}$ were plated onto 96-cell plates at the concentration of $1 \times 10^{5}$ cells $/ \mathrm{mL}$ and then cultured in $100 \mu \mathrm{L}$ basic culture medium. Twenty-four hours later, FTY720-P of different concentration ( $0 \mathrm{nM}, 1 \mathrm{nM}, 10 \mathrm{nM}$, and $100 \mathrm{nM})$ was added separately. $10 \mu \mathrm{L}$ CCK-8 solution was added 48 hours later and then measured the absorbance at $450 \mathrm{~nm}$. The fold change of proliferation rates was calculated based on the absorbance results and the rate in control group normalized to 1. As for proliferated neural stem sphere counting, FTY720$P$ was added into the medium and the number of stem spheres was calculated 48 hours later.

2.4. Identification of NSCs and Differentiated Neural Cells. After 30 min fixation in $4 \%$ paraformaldehyde, cells were washed three times with $0.01 \mathrm{M}$ PBS and incubated in $0.5 \%$ Triton-100. 1\% BSA was used prior to an overnight incubation with the primary antibodies. Anti-Brd-U (1:800, Sigma, USA), anti-Ki67 (1:200, Cell Signaling), and anti-Nestin ( $1: 400$, Millipore, USA) were used as stem cell markers. Anti$\beta$-tubulin III ( $1: 800$, Sigma, USA), anti-GFAP ( $1: 160$, Sigma, USA), anti-A2B5 (1:200, Abcam, USA), anti-Olig2 (1:500, Abcam, USA), and anti-CC1 $(1: 200$, Life Science Research, USA) were used as specific cell type makers. Anti-S1P1 (1:100, R\&D System, USA) or anti-S1P2 (1:200, Novus Biologicals,
USA) were used to confirm the expression of S1P receptors in NSCs. FITC-conjugated goat secondary antibodies $(1: 100$, Zhongshan, China) and TRITC-conjugated goat secondary antibodies $(1: 100$, Zhongshan, China) were used for labeling the different source of primary antibodies separately. Subsequently, the samples were stained with DAPI nuclei staining prior to confocal laser scanning microscopy (Leica, SP2, Germany).

2.5. Transwell (Boyden Chamber) Cell Migration Assay. In a total volume of $600 \mu \mathrm{L}$ basic culture medium containing FTY720-P was added at the 24-cell plates coated with polyL-lysine (Sigma, USA) while $100 \mu \mathrm{L}$ neurospheres cells were plated inside the Boyden Chamber at the density of $1 \times$ $10^{6}$ cells $/ \mathrm{mL}$ [17]. Eighteen hours later, the chambers were taken away, and the cells in each lower chamber were stained with crystal violet for the number analyzed.

2.6. Western Blot Analysis. The differentiated cells or NSCs were lysed (N-PER, Thermo Fisher, USA) and centrifuged at $14,0006 \times \mathrm{g}$ for 20 minutes. Protein concentration of the supernatant was determined using BCA protein assay kit (Beyotime Biotechnology, China). An equivalent amount of protein samples was loaded on SDS-polyacrylamide gels and transferred to PVDF membranes (Millipore, USA). The blots were blocked and subsequently probed with anti-GFAP ( $1: 400$, Sigma, USA), anti- $\beta$-tubulin III $(1: 1500$, Sigma, USA), anti-F-actin (Wanlei Biotechnology, China), and antiOlig2 (1:2000; Abcam) overnight. Secondary horseradish peroxidase-conjugated antibodies (1:5000; Zhongshan Biotechnology, China) were used and finally, blots were detected with enhanced chemiluminescence (ECL) detection reagents (Thermo Fisher, USA). $\beta$-Actin was recognized as the internal control in a parallel running. Bio-Rad Image Lab Software Version 5.2.1 or Image J Version 1.50i was used for optical densities quantification as we did previously [18]. Separate experiments were conducted for three times.

2.7. Statistical Analysis. The number of neurospheres was counted and averaged in a total of 5 systematic fields (same location of the 24-well plates in each group) under a $10 \mathrm{x}$ objective, and this was repeated five times independently. The percentage of differentiated cells were calculated as $\beta$-tubulin III, GFAP, CC1, or Olig2 positive, while GFAP positive astrocytes have two types based on their morphology, all divided by the total number of differentiated cells (DAPI-labeled) at 5 random fields under a 40x objective for each group in five independent experiments. Data were presented as mean \pm SD. Statistical analysis was performed using one-way analysis of variance (ANOVA) followed by Dunnett's multiple comparison tests. $P<0.05$ was considered to be statistically significant.

\section{Results}

3.1. Primary Cultured NSCs Have the Ability to Differentiate and Proliferate. Proliferating NSCs formed neurospheres in suspension on the following days, and they are Nestin (neural stem cell specific marker) positive (Figures 1(a) and 1(b)). Detection of DNA replication by Brd-U immunostaining of 


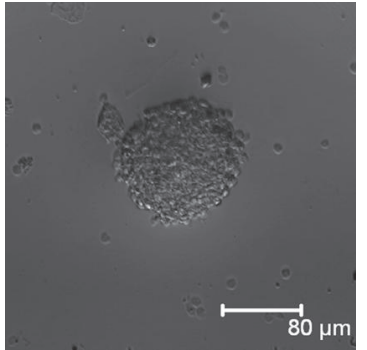

(a)

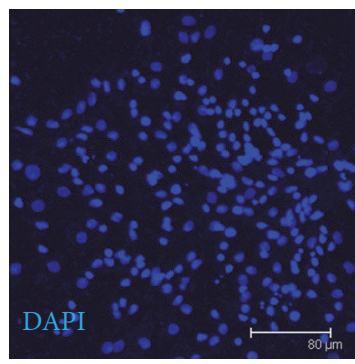

(e)

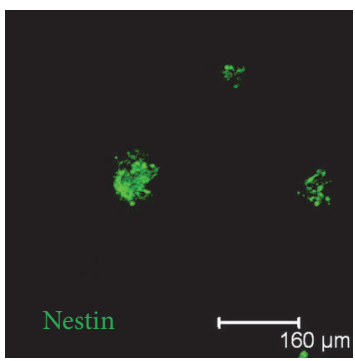

(b)

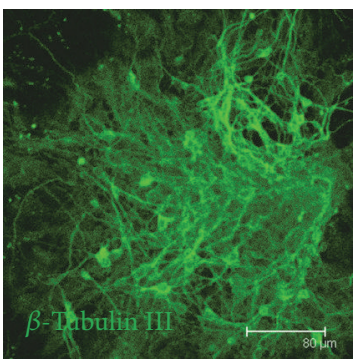

(f)

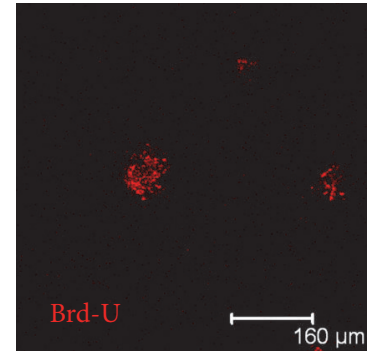

(c)

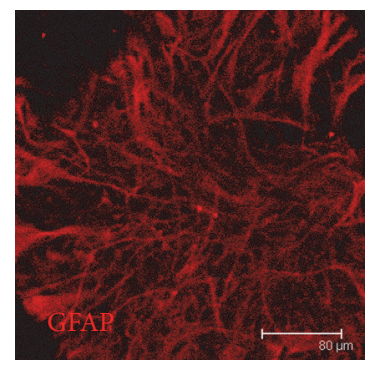

(g)

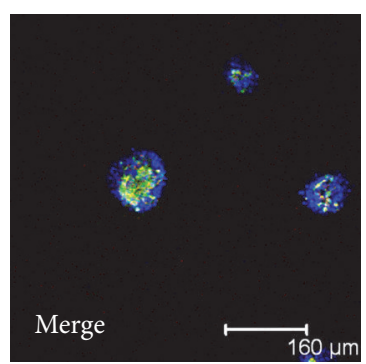

(d)

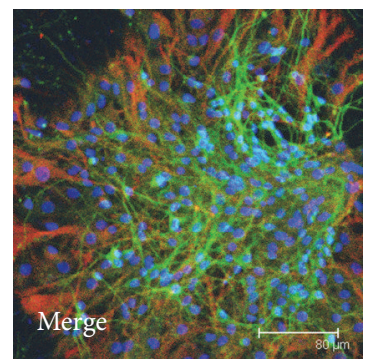

(h)

FIgURE 1: Identification of primary cultured NSCs in vitro. (a) Representative neurospheres in culture. ((b)-(d)) Immunocytochemical staining of purified NSCs with Nestin ((b), green) and Brd-U ((c), red) and merged with nucleus staining DAPI ((d), blue) (bar $=160 \mu \mathrm{m})$. ((e)-(h)) Nucleus staining ((e), blue) and immunocytochemical of differentiated astrocytes with $\beta$-tubulin III ((f), green) and GFAP ((g), red) and merged with (h) (bar $=80 \mu \mathrm{m})$.

the NSCs spheres confirmed that these cells undergo proliferation (Figure 1(c)). After withdrawal of bFGF and EGF and fetal bovine serum addition, the spheres differentiated into neurons and astrocytes as identified by staining of neuron-specific marker $\beta$-tubulin III and astrocyte-specific marker GFAP, which testified that the NSCs have multiple differentiation potentials (Figures $1(\mathrm{~g})$ and $1(\mathrm{~h})$ ). Besides, the neurospheres express S1P1 as it is double positive with Nestin (see Supplement Figure 1 in Supplementary Material available online at http://dx.doi.org/10.1155/2016/9671732).

3.2. FTY720 Promotes Proliferation of NSCs. There was an obvious increase in numbers of NSCs 48 hours after FTY720 intervention. What is more, some of the spheres were bigger in the 10 and 100 nM FTY720 groups compared to control and the $1 \mathrm{nM}$ FTY720 groups (Figures 2(a) and 2(b); Supplement Figure 2). The result was further confirmed by CCK-8 assay (Figure 2(c)). NSCs in the higher concentration groups had stronger proliferation ability, which is 1.45 - and 1.52 -fold increase compared to the control group, $P<0.05$. However, it seemed that FTY720 did not affect the proliferation rate of neural stem spheres at the concentration of $1 \mathrm{nM}$.

3.3. FTY720 Induces Differentiation of NSCs to Protoplasmic Astrocytes. The differentiating cells began to migrate out from the spheres 2 3 days later and developed morphological characteristics of neurons with spherical cell bodies and neurite-like processes or glial cells, including larger protoplasmic and slim fibrous outline astrocytes or short processes oligodendrocytes.
Differentiation of neurons and astrocytes was confirmed by immunocytochemical staining with specific marker $\beta$ tubulin III and GFAP (Figure 3(a)). Quantification analysis showed no neuron prefers differentiation of the NSCs because there were no significant differences in the number of neurons among groups, with $28.8 \pm 5.3 \%, 29.4 \pm 8.2 \%, 30.6 \pm$ $6.9 \%$, and $29.2 \pm 6.7 \%$ separately, $P>0.05$ (Figures $3(\mathrm{a}$ ) and $3(\mathrm{~b})$ ). Western blot analysis showed similar results (Figures $3(\mathrm{~d})$ and $3(\mathrm{e}))$.

However, the proportion of protoplasmic astrocytes obviously differs among groups. Larger cell body protoplasmic astrocytes (also known as type 1 astrocytes) were frequently observed in higher dose (10 nM and $100 \mathrm{nM}$ ) FTY720 groups, at $48.8 \pm 8.8 \%$ and $55.8 \pm 11.4 \%$, respectively, which increased significantly versus control and $1 \mathrm{nM}$ FTY720 group, with $6.8 \pm 4.3 \%$ and $10.2 \pm 4.3 \%$ separately, $P>0.05$ (Figures $3(\mathrm{a})$ and 3(c)). Meanwhile, the smaller fibrous astrocytes (also known as type 2 astrocytes) were more likely to be seen in the control and $1 \mathrm{nM}$ FTY720 group (data not shown). To determine whether it is the total number or only the relative frequency of protoplasmic astrocytes that increased, we next tested the protein level of GFAP using western blot. No differences of the relative GFAP protein level among groups were found, $P>0.05$ (Figures $3(\mathrm{f})$ and $3(\mathrm{~g})$ ).

To explore the differentiation of oligodendrocyte lineage, we stained the cells with Olig2 and CC1, and both were oligodendrocyte makers. There was a trend of increasing proportion of olig2 positive oligodendrocytes in the higher FTY720 groups, but lacking of significant differences, $P>0.05$ (Figures 4(a) and 4(b)). Nevertheless, more morphological matured oligodendrocytes were seen in higher concentration 

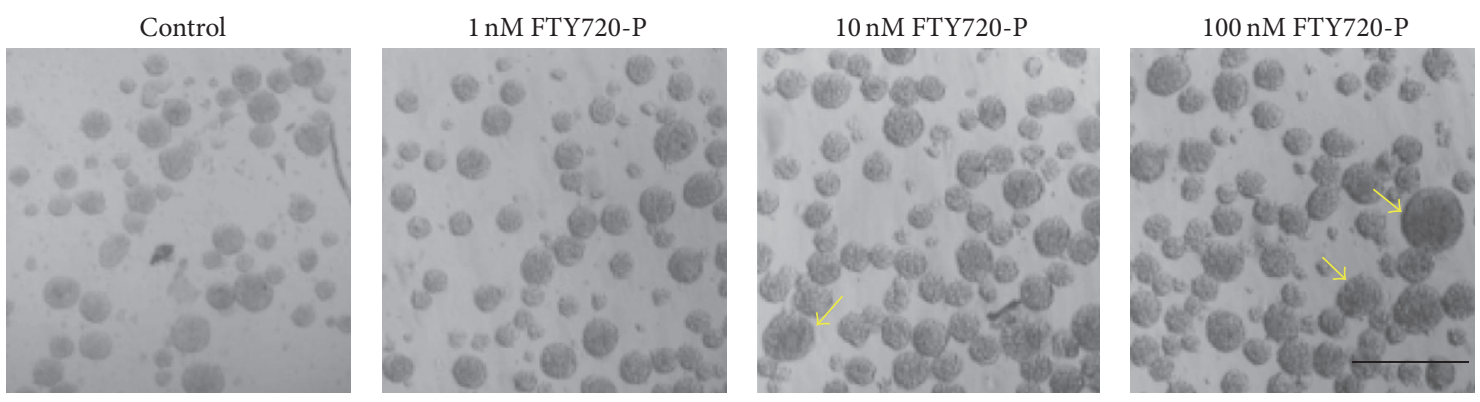

(a)

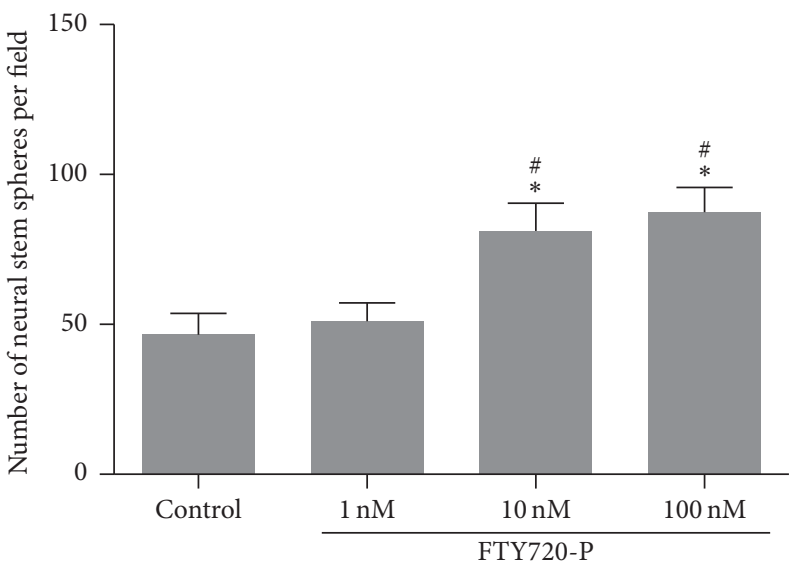

(b)

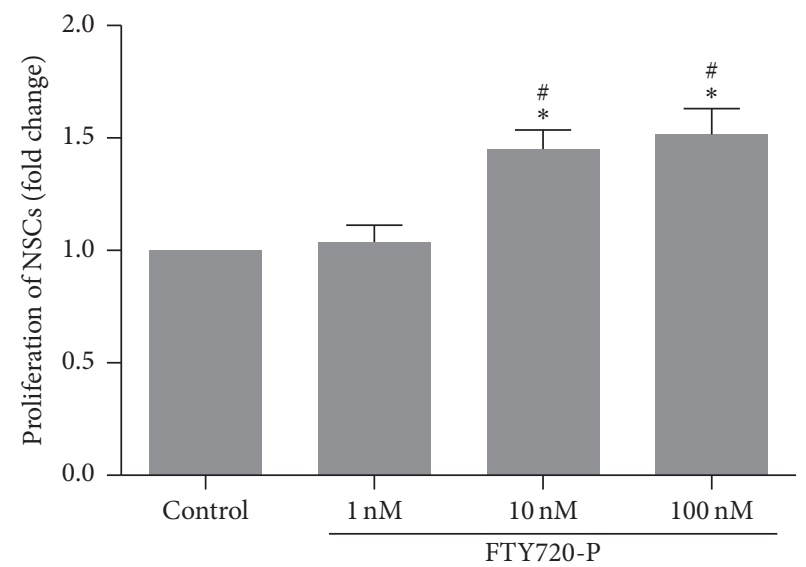

(c)

FIGURE 2: The proliferation of NSCs in the presence of FTY720. ((a)-(b)) Representative images of neurospheres and number quantification in response to FTY720. Bar $=500 \mu \mathrm{m}$. Arrows indicated lagers sizes of the neurospheres. (c) Proliferation rate indexes in the presence of FTY720. Fold changes in proliferation were calculated by the absorbance of $450 \mathrm{~nm}$ of CCK- 8 assay. Data represent the mean \pm SD; $n=5$ in each group; ${ }^{*} P<0.05$ compared with control group; ${ }^{\#} P<0.05$ compared with $1 \mathrm{nM}$ FTY720 group, by ANOVA followed by Dunnett's multiple comparison tests.

groups, and the proportion of CC1/Olig2 double positive cells was more in FTY groups (Figure 4(c)).

3.4. FTY720 Promotes Migration of NSCs. To assess the effects of FY720 on NSCs migration, Boyden Chamber and crystal violet staining were used in the experiment. The results showed promoted NSCs migration in a concentrationdependent manner 18 hours following FTY720 intervention. With the increase of FTY720 concentration, the number of cells migrating to the lower membrane of the chamber grows (Figure 5(a)). Significantly higher number of migrating cells were found in the $10 \mathrm{nM}$ and $100 \mathrm{nM}$ FTY720 groups (75.4 \pm 10.0 and $78.8 \pm 12.7$, resp.) in comparison with control (47.6 \pm $6.4, P=0.001$ ). The $1 \mathrm{nM}$ FTY720 group showed a little higher but no significant increase in the number of migrating cells $(56.8 \pm 9.2, P>0.05$ compared with control group) (Figure 5(b)).

Previous studies reported that filamentous actin (F-actin) polymerization is required for ligand-induced cell chemotaxis [19]. We then examined the protein expression level of F-actin in the NSCs. Consistent with the result in Boyden Chamber assay, F-actin was evidently upregulated in NSCs stimulated with $10 \mathrm{nM}$ and $100 \mathrm{nM}$ FTY720, $P<0.05$, compared with control group (Figures 5(c) and 5(d)).

\section{Discussion}

In the present study, we tested the pluripotency and the expression of S1P1 of brain-derived NSCs. Moreover, these cells keep the ability of proliferation as we did before $[18,20]$. Our data demonstrated that FTY720 could promote proliferation, differentiation of protoplasmic astrocytes, and migration of NSCs in a dose-dependent manner, because most of these effects were shown in higher concentration groups. Additionally, we found out that there were more matured oligodendrocytes on the FTY intervention groups.

Harada and his colleagues examined the effects of S1P on neural progenitor cells and reported that S1P induced proliferation of neural progenitor cells via S1P receptors $(\mathrm{S} 1 \mathrm{P} 1 / 3)$ activation and phosphorylation of extracellular signal-regulated kinase (ERK) by Brd-U incorporation assay [21]. This opinion was supported by another study about embryonic neural stem cells. However, other studies on lymphocytes and astrocytes have indicated the S1P-analog FTY720 plays a dual role in activation or inhibition on S1P1 $[9,22]$. Therefore, the role of FTY720 on NSCs might not be the same with endogenous S1P.

Studies have shown that FTY720 increases the viability and neurogenic potential of irradiated NSCs of the hippocampus $[23,24]$. For example, Stessin et al. reported more 
DAPI

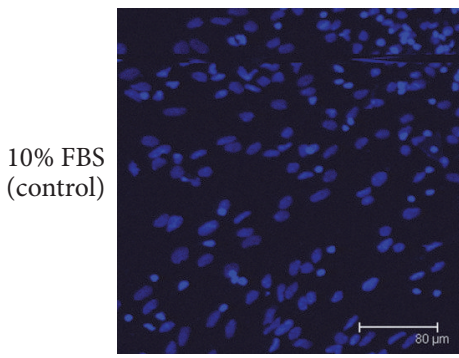

1 nM FTY720-P + $10 \%$ FBS

10 nM FTY720-P + $10 \%$ FBS

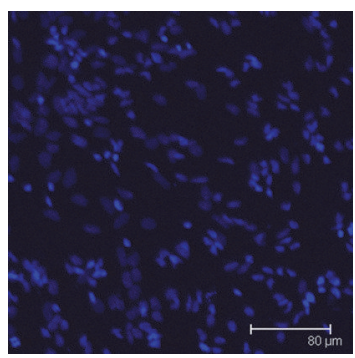

GFAP
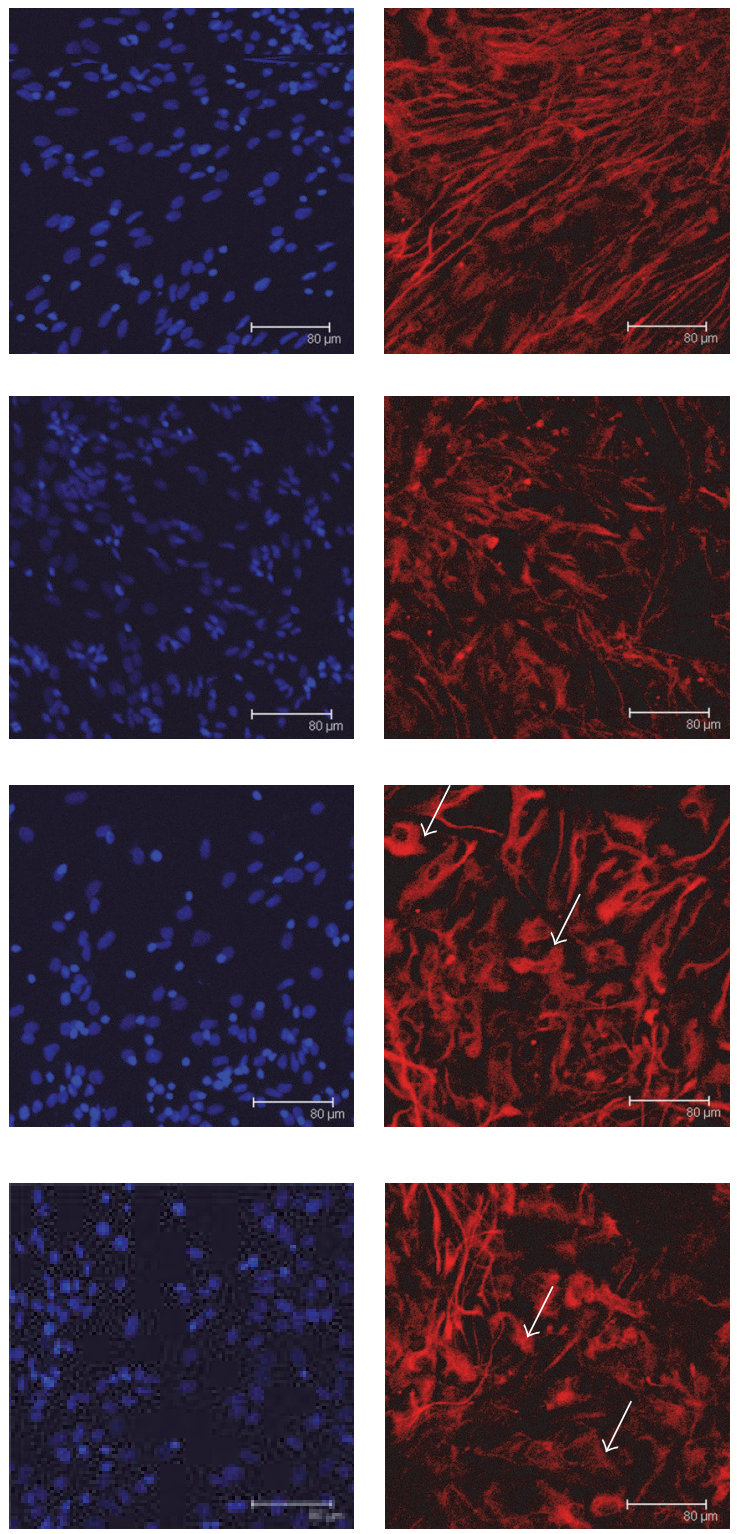

(a)
$\beta$-Tubulin III
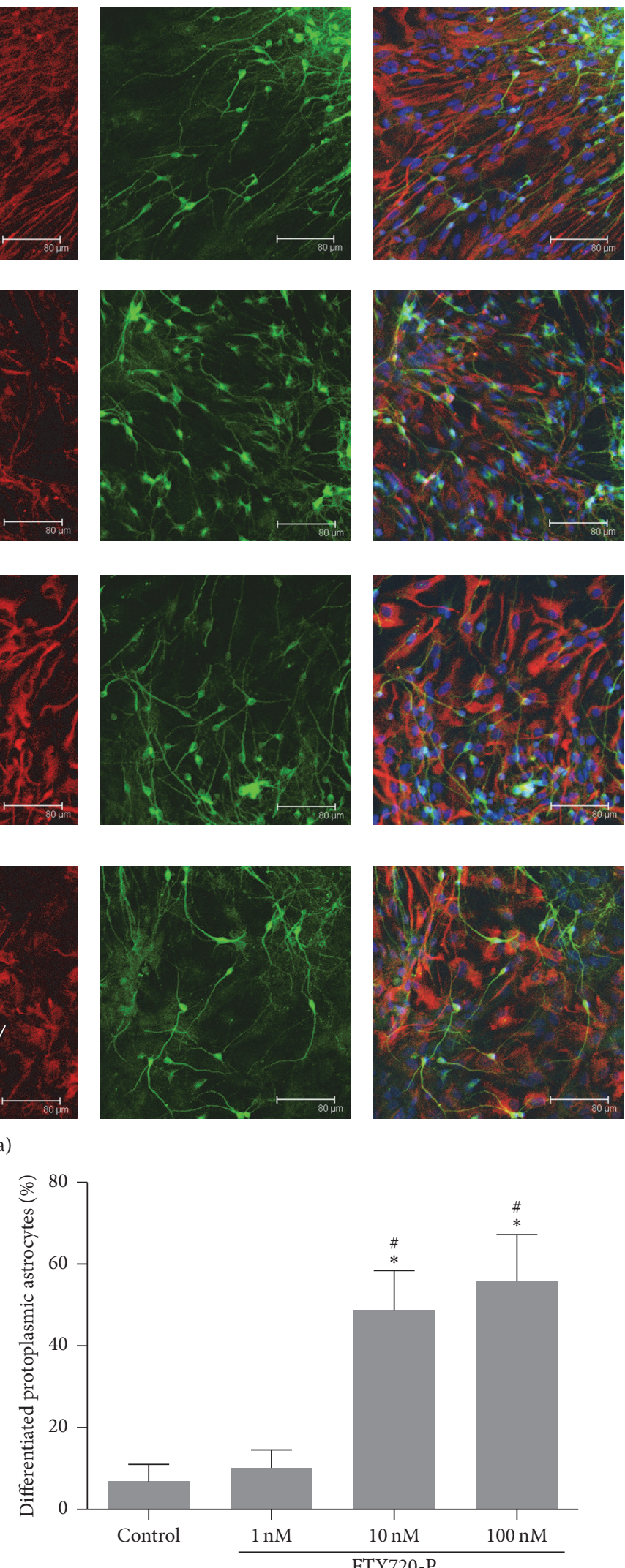

(c) (b)

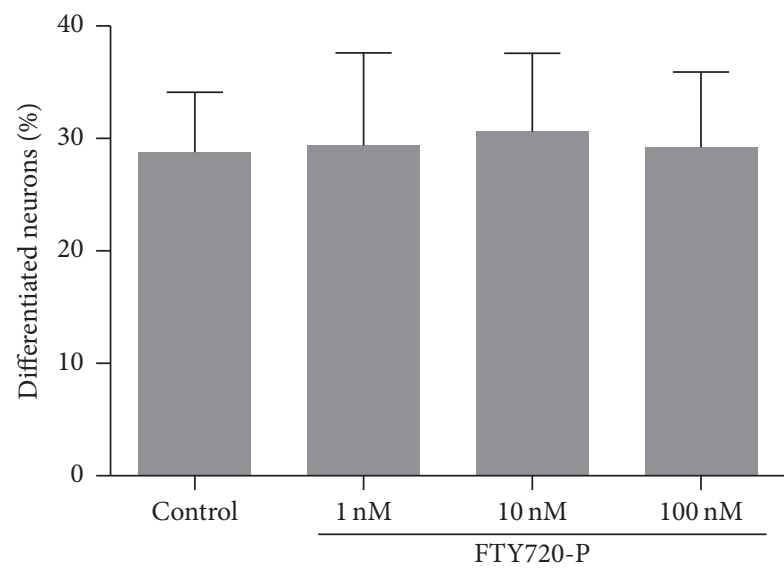

Figure 3: Continued. 


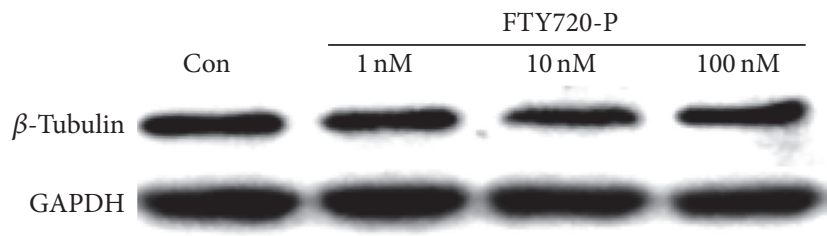

(d)

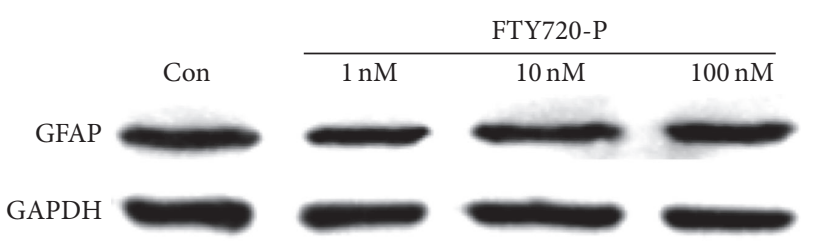

(f)

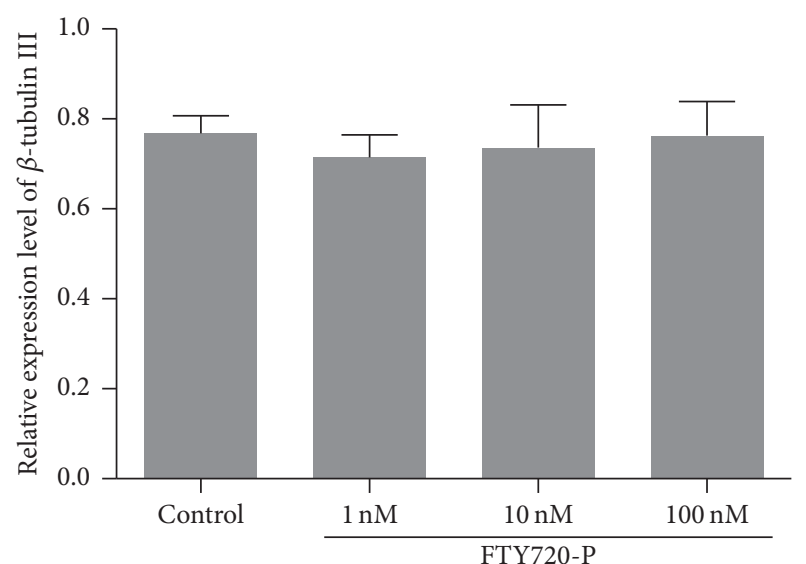

(e)

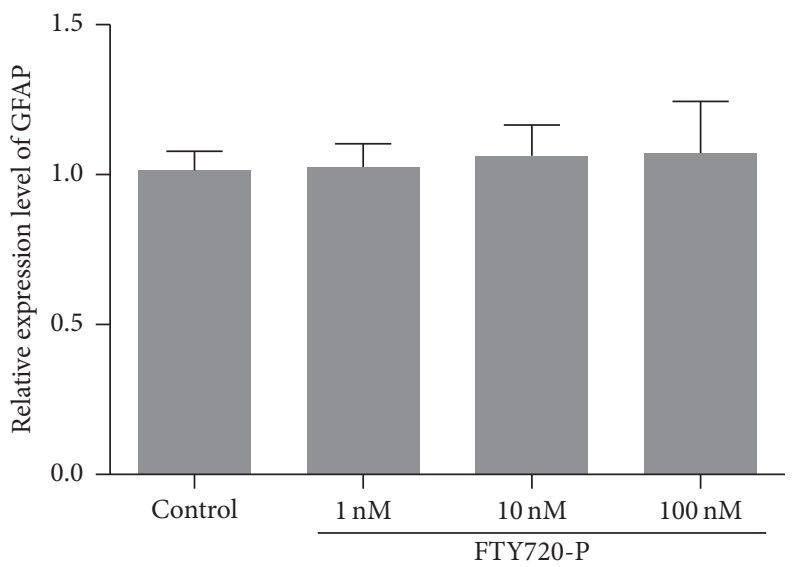

(g)

FIGURE 3: Differentiation of NSCs into neurons and astrocytes in the presence of FTY720. (a) Representative images of neurons ( $\beta$-tubulin III positive, in green) and astrocytes (GFAP positive, in red) in different groups. Bar $=80 \mu \mathrm{m}$. Arrows indicate protoplasmic astrocytes. ((b)-(c)) Quantification of the proportion of differentiated neurons and astrocytes. $n=5$. ((d)-(e)) Western blot analysis of $\beta$-tubulin III protein in different groups. $n=3$ in each group. ((f)-(g)) Western blot analysis of GFAP protein in different groups. $n=3$ in each group. ${ }^{*} P<0.05$ compared with control group; ${ }^{*} P<0.05$ compared with $1 \mathrm{nM}$ FTY720 group, by ANOVA followed by Dunnett's multiple comparison tests.

neuronal (NeuN positive) differentiation in the presence of $10 \mathrm{nM}$ FTY720 in the irradiated brain, which counteracted the radiation-induced suppression of NSCs neurogenic potential. However, we failed to observe similar results in cultured NSCs, as no significant differences were found among groups of $\beta$-tubulin III positive cells. Our conclusion was supported by a study on a viral-induced demyelination mice model [16]. And no neuron differentiation on embryonic hippocampal NSCs has also been reported recently [25].

Surprisingly, the number of type- 2 astrocytes, also known as the protoplasmic astrocyte, was much more in higher concentrations of FTY720, while the total amount of GFAP protein levels was not affected. Similar results have shown recently that the 3 major cell types differentiation were not affected by FTY using western blot [25]. Therefore, we speculate that FTY720 might be able to keep a relatively "stable differentiation model" of NSCs. Although coculture of protoplasmic astrocytes with NSCs promotes differentiation of neurons [15], S1PRs in protoplasmic astrocytes might be negatively regulated by FTY720 [9], resulting in suppression effects on neuronal differentiation, which might be another explanation why we did not observe neuronal preferences. However, as we know that protoplasmic astrocytes are mainly distributed in gray matter and participate in the formation of the blood-brain barrier, they are considered to be a benefit in CNS repair. Therefore, it would be one of the possible mechanisms of FTY720's neuroprotective effects in CNS disorders [26, 27]. Evidence about whether FTY have the ability to promote the development of oligodendrocytes remains controversial $[28,29]$. Here, we found no induction of more oligodendrocyte lineage cells by FTY treated NSCs. However, more morphological matured oligodendrocytes were seen in the higher dose FTY720 groups. The different results among groups might be because of people using different originated NSCs or diseases models [1, 16, 29-31].

Migration ability is one of the premises of NSCs in repairing CNS injuries. In this study, FTY720 stimulates chemoattractant activity of NSCs from the upper chamber to the lower in Transwell assay. The migration activity seems to be strengthening with FTY720 concentration increase. This 


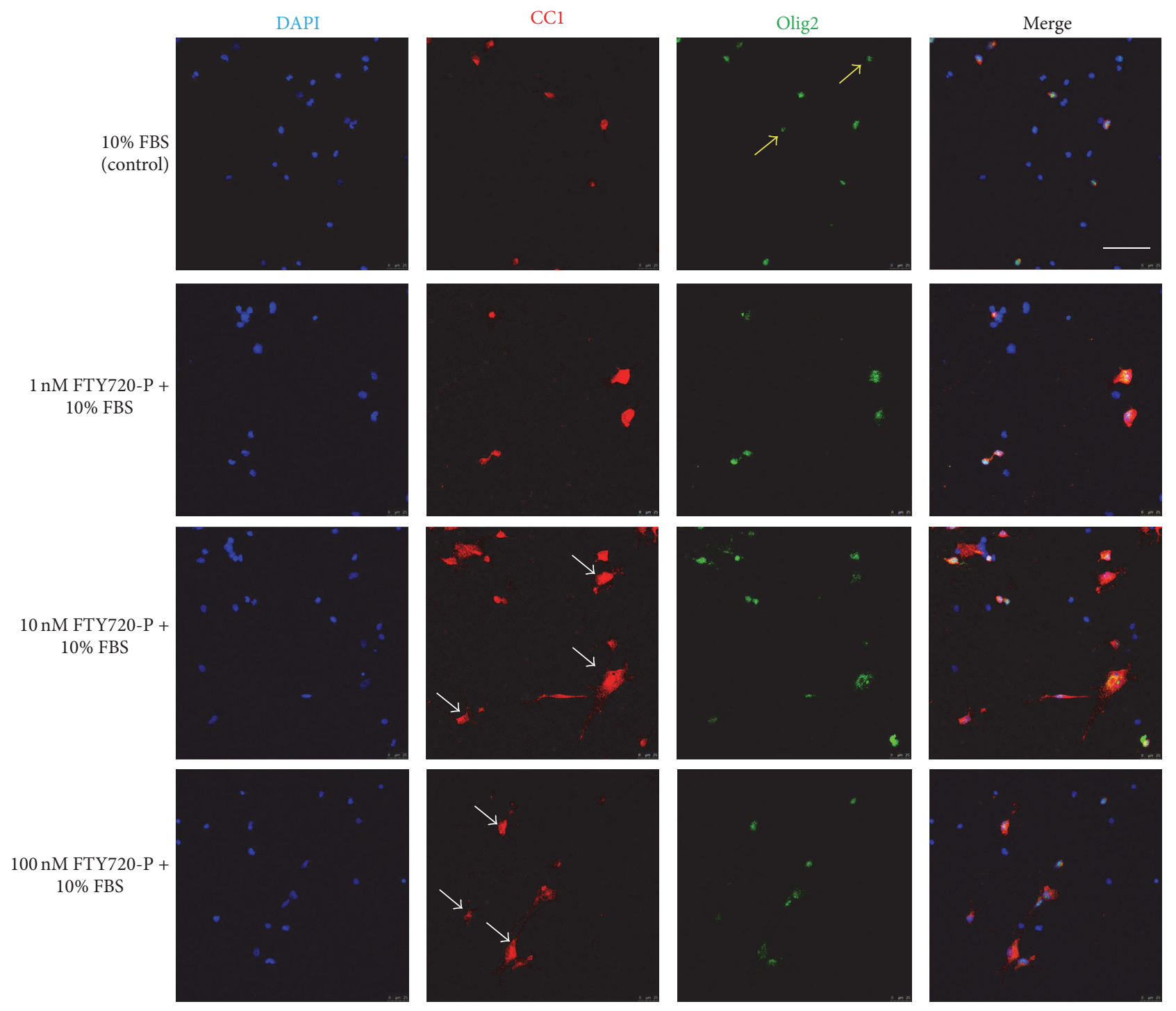

(a)

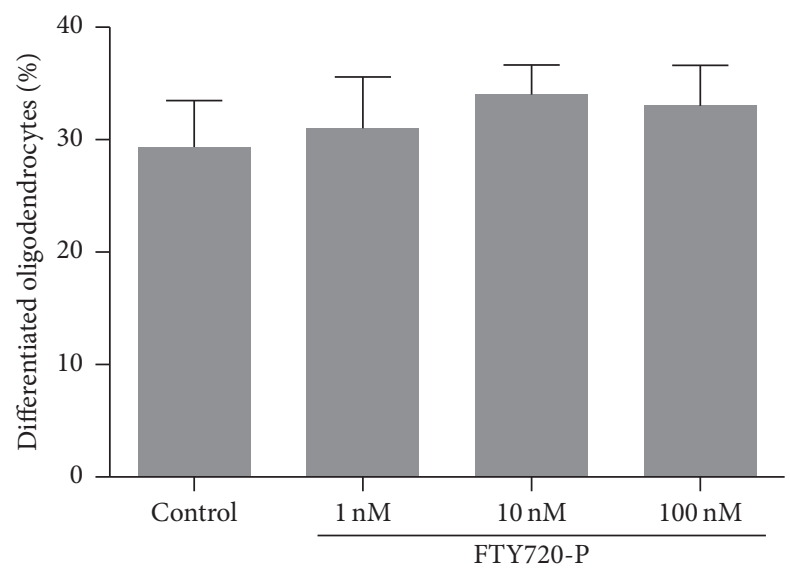

(b)

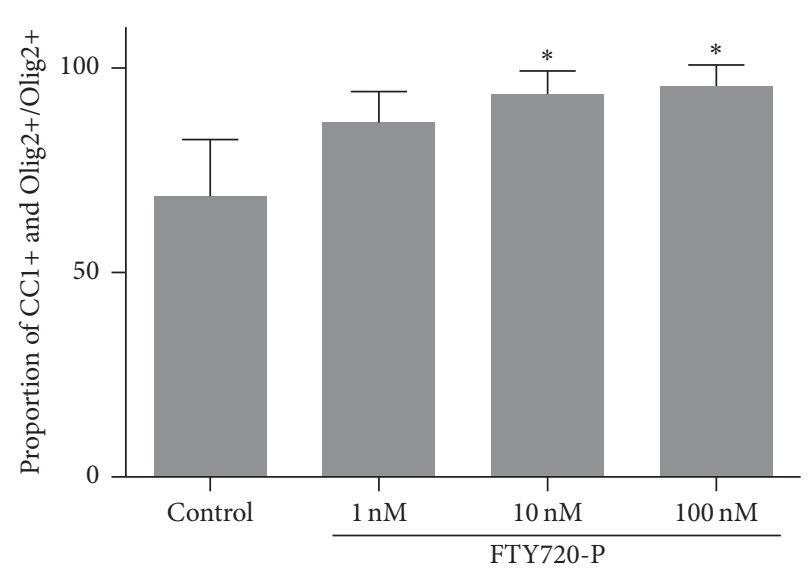

(c)

FIGURE 4: Differentiation of NSCs into oligodendrocytes in the presence of FTY720. (a) Representative images of differentiated oligodendrocytes in different groups. Matured oligodendrocytes are both Olig2 (Green) and CC1 (Red) positive. Parts of the Olig2+ cells in the control group did not express CC1 (yellow arrows) while more morphological matured (white arrows) oligodendrocytes were seen in higher FTY720 concentration groups. Bar $=50 \mu \mathrm{m}$. ((b)-(c)) Quantification of the proportion of differentiated oligodendrocytes. $n=3$ in each group. ${ }^{*} P<0.05$, compared with control group, by ANOVA, followed by Dunnett's multiple comparison tests. 


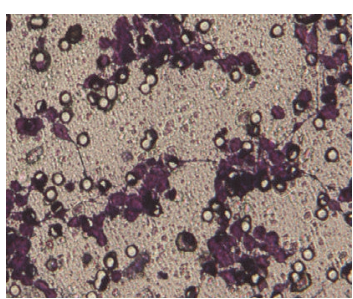

Control

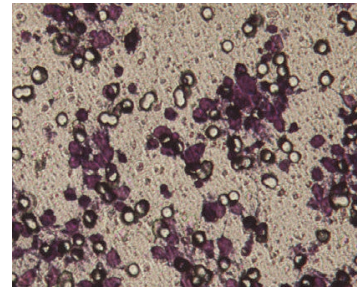

1 nM FTY720-P

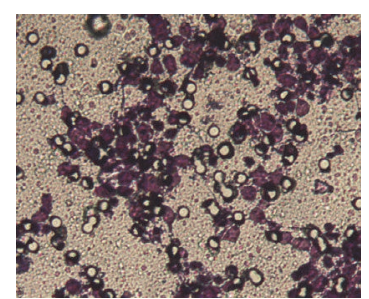

10 nM FTY720-P

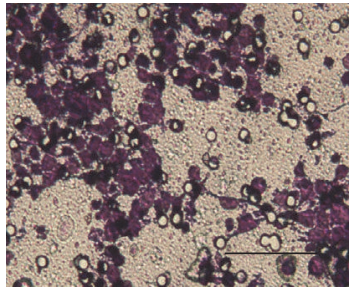

100 nM FTY720-P

(a)

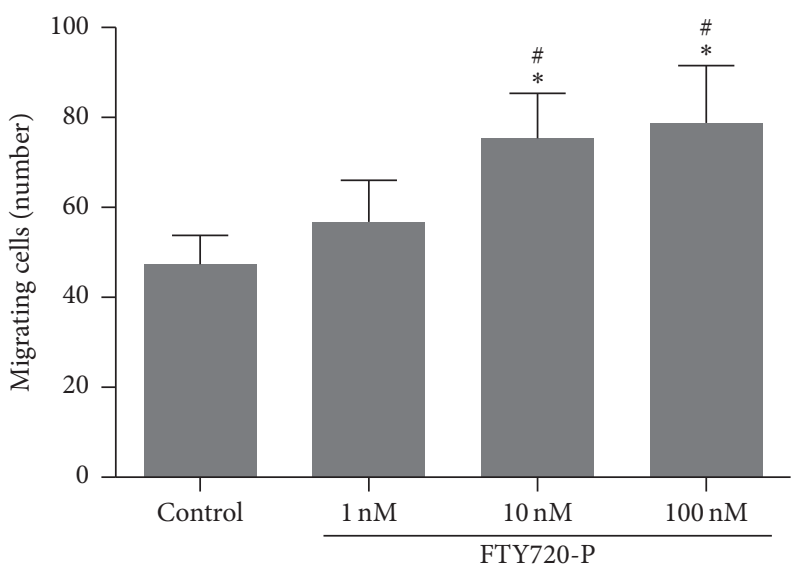

(b)

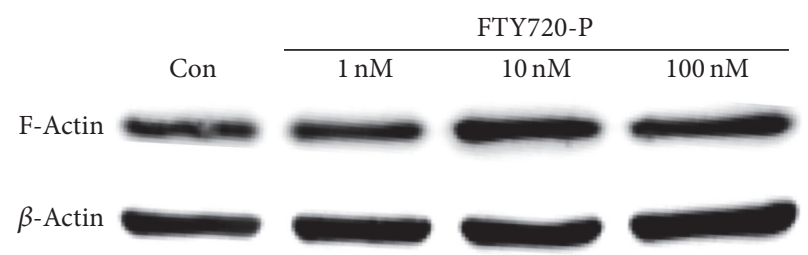

(c)

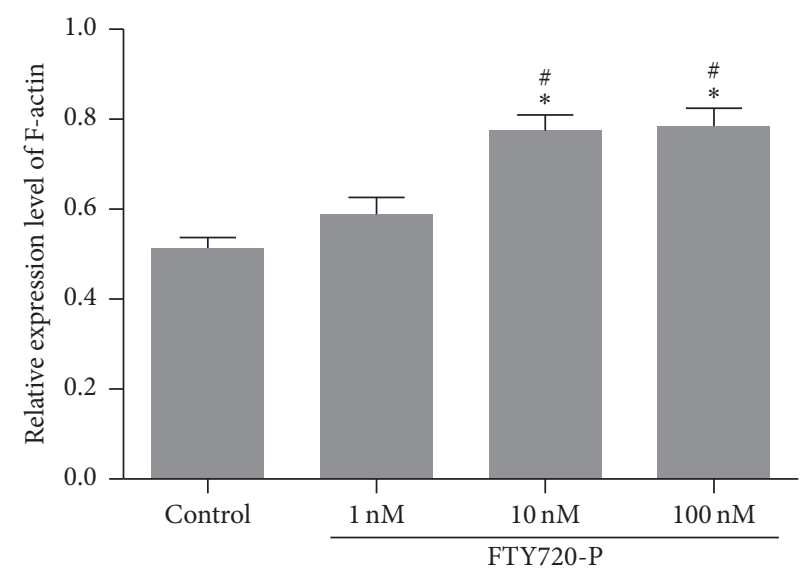

(d)

FIGURE 5: Quantification analysis of migrated cell numbers with FTY720 intervention. ((a)-(b)) Number of migrated cells from upper Boyden Chamber with crystal violet staining (bar $=100 \mu \mathrm{m})$. Migrated cells were counted by crystal violet staining and averaged three random microscope fields of each sample; five independent experiments were done. ((c)-(d)) Relative expression level of F-actin among groups. Mean \pm SD and $n=5$ in each group. ${ }^{*} P<0.05$ compared with control group; ${ }^{\#} P<0.05$ compared with 1 nM FTY720 group, by ANOVA followed by Dunnett's multiple comparison tests.

was further verified by protein level of filamentous actin (Factin). Similar results were found in NSCs transplantation studies because inhibition of S1P1/3 abolishes the chemoattractant activity of S1P/S1P receptors [17, 32]. Although activation of S1P2 would reduce migration according to Kimura's research, no reports mentioned FTY720 could combine this receptor $[21,32]$. As there are multiple targets downstream FTY720/S1PRs, it is not clear which signal pathway underlies the mechanism. Recently, Kassmer et al. reported that the migratory response of germline progenitor cells is dependent on activation of PI3K and Racl downstream of the S1P1 [33]. This G-protein-coupled receptors mediate cellular responses to attractive chemokines in a variety of cell types, including NSCs.

Some inadequacies need to be improved in further work. On the one hand, molecular pathways underlying the NSCs biological behavior in the presence of FTY720 were not tested. On the other hand, in vivo experiments need to be introduced to the present study. Though it is not certain whether FTY720's neuroprotective effects have correlations 
with NSCs, we believe that the application of this drug will be confined not only to MS but also to CNS injuries and other degenerative diseases.

\section{Competing Interests}

No conflicts of interest are declared.

\section{Acknowledgments}

This work was supported by grants from the National Natural Science Foundation of China (no. 81171859), Chongqing National Natural Science Fund (CSTC, no. 2012jjA10058), and Scientific and Technological Research Program of Chongqing Municipal Education Commission (Grant no. KJ1500237). The authors gratefully acknowledge financial support from China Scholarship Council. The funder had role in study design, data collection, and analysis, decision to publish, and preparation of the manuscript.

\section{References}

[1] L. Harris, O. Zalucki, M. Piper, and J. I.-T. Heng, "Insights into the biology and therapeutic applications of neural stem cells," Stem Cells International, vol. 2016, Article ID 9745315, 18 pages, 2016.

[2] N. Zhang, J. Wimmer, S.-J. Qian, and W.-S. Chen, "Stem cells: current approach and future prospects in spinal cord injury repair," Anatomical Record, vol. 293, no. 3, pp. 519-530, 2010.

[3] B. T. Tan, J. Yu, Y. Yin, G. W. Jia, W. Jiang, and L. H. Yu, “The Olig family affects central nervous system development and disease," Neural Regeneration Research, vol. 9, no. 3, pp. 329-336, 2014.

[4] R. Covacu and L. Brundin, "Effects of neuroinflammation on neural stem cells," The Neuroscientist, 2015.

[5] H. Ulrich, M. P. Abbracchio, and G. Burnstock, "Extrinsic purinergic regulation of neural stem/progenitor cells: implications for CNS development and repair," Stem Cell Reviews and Reports, vol. 8, no. 3, pp. 755-767, 2012.

[6] R. E. Toman and S. Spiegel, "Lysophospholipid receptors in the nervous system," Neurochemical Research, vol. 27, no. 7-8, pp. 619-627, 2002.

[7] Y. A. Hannun and L. M. Obeid, "Principles of bioactive lipid signalling: lessons from sphingolipids," Nature Reviews Molecular Cell Biology, vol. 9, no. 2, pp. 139-150, 2008.

[8] I. Fischer, C. Alliod, N. Martinier, J. Newcombe, C. Brana, and S. Pouly, "Sphingosine kinase 1 and sphingosine 1-phosphate receptor 3 are functionally upregulated on astrocytes under proinflammatory conditions," PLOS ONE, vol. 6, no. 8, Article ID e23905, 2011.

[9] C. Wu, S. Y. Leong, C. S. Moore et al., "Dual effects of daily FTY720 on human astrocytes in vitro: relevance for neuroinflammation," Journal of Neuroinflammation, vol. 10, article 41, 2013.

[10] H. Noda, H. Takeuchi, T. Mizuno, and A. Suzumura, "Fingolimod phosphate promotes the neuroprotective effects of microglia," Journal of Neuroimmunology, vol. 256, no. 1-2, pp. 13-18, 2013.

[11] T. C. Spohr, R. S. Dezonne, J. Nones et al., "Sphingosine 1phosphate-primed astrocytes enhance differentiation of neuronal progenitor cells," Journal of Neuroscience Research, vol. 90, no. 10, pp. 1892-1902, 2012.
[12] R. Sánchez-Sánchez, S. L. Morales-Lázaro, J.-M. Baizabal, M. Sunkara, A. J. Morris, and D. Escalante-Alcalde, "Lack of lipid phosphate phosphatase-3 in embryonic stem cells compromises neuronal differentiation and neurite outgrowth," Developmental Dynamics, vol. 241, no. 5, pp. 953-964, 2012.

[13] J. Chun and H.-P. Hartung, "Mechanism of action of oral fingolimod (FTY720) in multiple sclerosis," Clinical Neuropharmacology, vol. 33, no. 2, pp. 91-101, 2010.

[14] X. Liu, Y. Zhu, and W. Gao, "Isolation of neural stem cells from the spinal cords of low temperature preserved abortuses," Journal of Neuroscience Methods, vol. 157, no. 1, pp. 64-70, 2006.

[15] Y. Liu, L. Wang, Z. Long, L. Zeng, and Y. Wu, "Protoplasmic astrocytes enhance the ability of neural stem cells to differentiate into neurons in vitro," PLOS ONE, vol. 7, no. 5, Article ID e38243, 2012.

[16] C. A. Blanc, J. J. Grist, H. Rosen, I. Sears-Kraxberger, O. Steward, and T. E. Lane, "Sphingosine-1-phosphate receptor antagonism enhances proliferation and migration of engrafted neural progenitor cells in a model of viral-induced demyelination," The American Journal of Pathology, vol. 185, no. 10, pp. 2819-2832, 2015.

[17] A. Kimura, T. Ohmori, Y. Kashiwakura et al., "Antagonism of sphingosine 1-phosphate receptor-2 enhances migration of neural progenitor cells toward an area of brain infarction," Stroke, vol. 39, no. 12, pp. 3411-3417, 2008.

[18] B. T. Tan, L. Wang, S. Li et al., "Retinoic acid induced the differentiation of neural stem cells from embryonic spinal cord into functional neurons in vitro," International Journal of Clinical and Experimental Pathology, vol. 8, pp. 8129-8135, 2015.

[19] G. Wang, L. Zhang, X. Chen et al., "Formylpeptide receptors promote the migration and differentiation of rat neural stem cells," Scientific Reports, vol. 6, Article ID 25946, 2016.

[20] Y. Liu, R.-R. Liu, L. Wang, L. Zeng, Z.-Y. Long, and Y.-M. Wu, "The effects of different phenotype astrocytes on neural stem cells differentiation in co-culture," Neuroscience Letters, vol. 508, no. 2, pp. 61-66, 2012.

[21] J. Harada, M. Foley, M. A. Moskowitz, and C. Waeber, "Sphingosine-1-phosphate induces proliferation and morphological changes of neural progenitor cells," Journal of Neurochemistry, vol. 88, no. 4, pp. 1026-1039, 2004.

[22] M. Matloubian, C. G. Lo, G. Cinamon et al., "Lymphocyte egress from thymus and peripheral lymphoid organs is dependent on S1P receptor 1," Nature, vol. 427, no. 6972, pp. 355-360, 2004.

[23] A. M. Stessin, D. B. Gursel, A. Schwartz et al., "FTY720, sphingosine 1-phosphate receptor modulator, selectively radioprotects hippocampal neural stem cells," Neuroscience Letters, vol. 516, no. 2, pp. 253-258, 2012.

[24] A. M. Stessin, C. Sison, J. Nieto, M. Raifu, and B. Li, “The role of postoperative radiation therapy in the treatment of Meningeal hemangiopericytoma-experience from the SEER database," International Journal of Radiation Oncology Biology Physics, vol. 85, no. 3, pp. 784-790, 2013.

[25] Y. Sun, F. Hong, L. Zhang, and L. Feng, "The sphingosine-1phosphate analogue, FTY-720, promotes the proliferation of embryonic neural stem cells, enhances hippocampal neurogenesis and learning and memory abilities in adult mice," British Journal of Pharmacology, vol. 173, no. 18, pp. 2793-2807, 2016.

[26] Y. Norimatsu, T. Ohmori, A. Kimura et al., "FTY720 improves functional recovery after spinal cord injury by primarily nonimmunomodulatory mechanisms," The American Journal of Pathology, vol. 180, no. 4, pp. 1625-1635, 2012. 
[27] F. S. Hoffmann, J. Hofereiter, H. Rübsamen et al., "Fingolimod induces neuroprotective factors in human astrocytes," Journal of Neuroinflammation, vol. 12, article 184, 2015.

[28] J. Zhang, Z. G. Zhang, Y. Li et al., "Fingolimod treatment promotes proliferation and differentiation of oligodendrocyte progenitor cells in mice with experimental autoimmune encephalomyelitis," Neurobiology of Disease, vol. 76, pp. 57-66, 2015.

[29] M. N. Alme, A. E. Nystad, L. Bø et al., "Fingolimod does not enhance cerebellar remyelination in the cuprizone model," Journal of Neuroimmunology, vol. 285, pp. 180-186, 2015.

[30] Q. L. Cui, J. Fang, T. E. Kennedy, G. Almazan, and J. P. Antel, "Role of p38MAPK in S1P receptor-mediated differentiation of human oligodendrocyte progenitors," Glia, vol. 62, no. 8, pp. 1361-1375, 2014.

[31] A. Yazdi, H. Baharvand, and M. Javan, "Enhanced remyelination following lysolecithin-induced demyelination in mice under treatment with fingolimod (FTY720)," Neuroscience, vol. 311, pp. 34-44, 2015.

[32] A. Kimura, T. Ohmori, R. Ohkawa et al., "Essential roles of sphingosine 1-phosphate/S1P1 receptor axis in the migration of neural stem cells toward a site of spinal cord injury," Stem Cells, vol. 25, no. 1, pp. 115-124, 2007.

[33] S. H. Kassmer, D. Rodriguez, A. D. Langenbacher, C. Bui, and A. W. De Tomaso, "Migration of germline progenitor cells is directed by sphingosine-1-phosphate signalling in a basal chordate," Nature Communications, vol. 6, article 8565, 2015. 

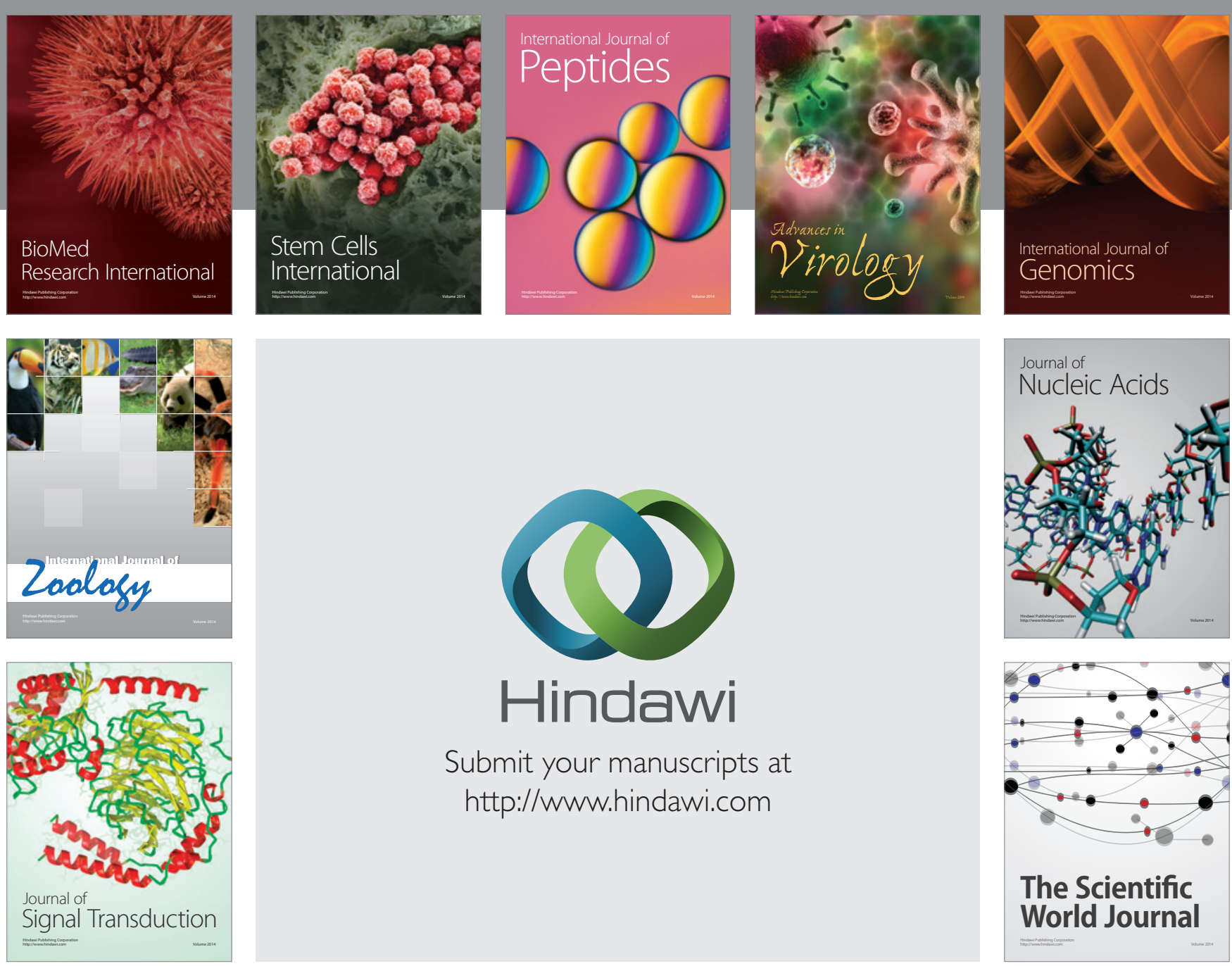

Submit your manuscripts at

http://www.hindawi.com
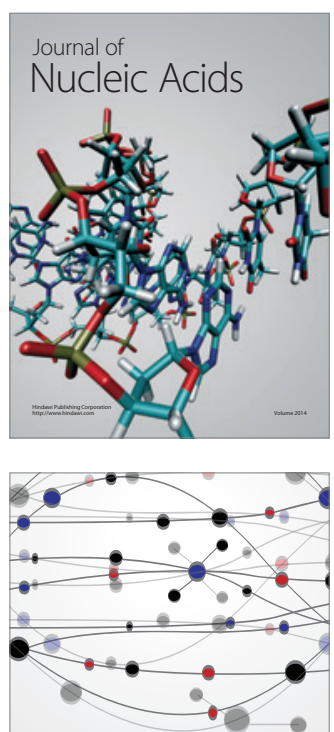

The Scientific World Journal
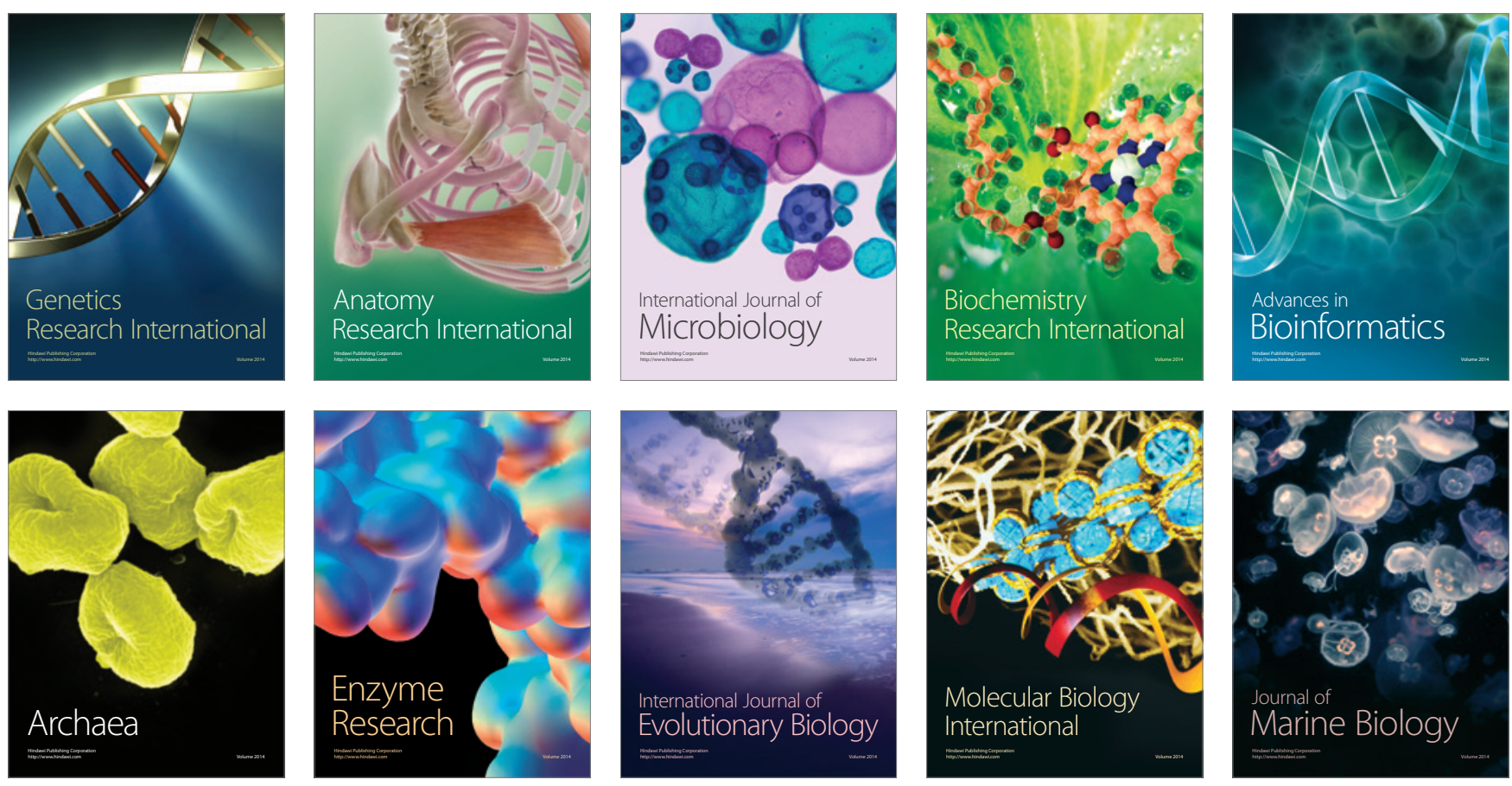